\title{
Renaturation of Lyophilized Concanavalin a Treated in Water Content Controlled Hydrated Ionic Liquids
}

\author{
Kyoko Fujita *, Ryota Fujii and Kimiyoshi Ichida
}

\author{
Department of Pathophysiology, Tokyo University of Pharmacy and Life Sciences, 1432-1 Horinouchi, Hachioji, \\ Tokyo 192-0392, Japan; y151159@toyaku.ac.jp (R.F.); ichida@toyaku.ac.jp (K.I.) \\ * Correspondence: kyokof@toyaku.ac.jp; Tel.: +81-42-676-5453
}

\begin{abstract}
A fraction of commercially available lyophilized concanavalin A (ConA) shows low mannose binding activity. In this study, we investigated the effects of the component ion and water content of hydrated ionic liquids (ILs) as treatment solvent on the mannose binding ability of lyophilized ConA. While high mannose binding ability was observed treated in hydrated ILs composed of dihydrogen phosphate anion with three water molecules per ion pair, it decreased with increase in water content. In contrast, no increase in mannose binding ability was observed after treatment with hydrated ILs composed of bromide anions. A steady response in mannose binding was observed after treatment with hydrated cholinium dihydrogen phosphate ([ch][dhp]). Following treatment with hydrated [ch][dhp] with three water molecules per ion pair, the mannose binding ability of lyophilized ConA increased twofold, while the low and high mannose binding ConA fraction showed an increase of 3.3 and 1.8 in the binding response, respectively.
\end{abstract}

Keywords: hydrated ionic liquids; renaturation; water content; mannose binding ability

check for

updates

Citation: Fujita, K.; Fujii, R.; Ichida, K. Renaturation of Lyophilized Concanavalin a Treated in Water Content Controlled Hydrated Ionic Liquids. Appl. Sci. 2021, 11, 57. https://dx.doi.org/10.3390/app1101 0057

Received: 26 November 2020 Accepted: 18 December 2020 Published: 23 December 2020

Publisher's Note: MDPI stays neutral with regard to jurisdictional claims in published maps and institutional affiliations.

Copyright: () 2020 by the authors. Licensee MDPI, Basel, Switzerland. This article is an open access article distributed under the terms and conditions of the Creative Commons Attribution (CC BY) license (https: / / creativecommons.org / licenses/by/4.0/).

\section{Introduction}

Lyophilization, also known as freeze-drying or cryodesiccation, is a dehydration process performed under low-temperature and -pressure conditions. Freeze-drying yields high-quality products because there is minimal thermal damage [1]. Lyophilization has become a standard practice in the life science industry because lyophilized products retain their original properties; by adding water or a suitable solvent, they can be easily and quickly reconstituted. However, some biomolecules like serum, vaccines, and proteins may get damaged by the stress associated with freezing, freeze-drying, or both. Thus, the activity of biomolecules after lyophilization often decreases, in comparison to nonlyophilized materials [2]. The development of methods aiming for the renaturation and recovery of the activity of lyophilized biomolecules is necessary to extend the shelf-life of these macromolecules.

Ionic liquids (ILs) are salts with melting points below $100{ }^{\circ} \mathrm{C}$. The properties of ILs can be changed by ensuring the appropriate structural design of their component ions. ILs have been gathering attention as green [3], multi-use reaction media [4,5] and solvents for a variety of applications. For example, ILs have been studied as potential candidates for substituents of reaction media in electrochemistry [6], enzymatic catalysis [7,8], and green chemistry [9] because of their excellent stability [10] and unusual solvent properties [11-14]. They have also been investigated for their potential to assist renaturation of proteins [15]. Some ILs, e.g., triethyl ammonium phosphate and imidazolium type cation with halide anion, have shown to be as effective as other small molecular additives when used as refolding agents in aqueous phase $[16,17]$. On the other hand, hydrated ILs, which are a mixture of ILs and a small amount of water, have showed great potential as solvents for the dissolution and refolding of aggregated and inactive proteins $[18,19]$. By selecting the suitable component ion and water content, inclusion bodies and thermally aggregated proteins can be dissolved and subsequently refolded in hydrated ILs. 
In this study, we investigated the effect of hydrated ILs as a treatment solvent for the renaturation of lyophilized inactive biomolecules. ConA has been selected as the target biomolecule to assess the capability of hydrated ILs on the recovery of protein functionality. ConA is a type of lectin with a specific affinity for mannose and glucose. In the past, ConA has been used in the medical field as a lymphocyte transformation test, and as a tumor marker [20]. We have previously reported the improvement in the shelf life and thermal stability of proteins (including ConA) after dissolution in hydrated ILs, especially in cholinium dihydrogen phosphate ([ch][dhp]) [21,22]. Furthermore, thermally aggregated ConA has shown a greater solubility and refolding behavior in some selected hydrated ILs [18]. Hydrated ILs composed of kosmotropic anions, such as dihydrogen phosphate anions, have been reported to be effective at dissolving proteins and maintaining their original higher-order structure [23]. In this study, we prepared several ILs composed of different cations and dhp anion or bromide anion (Figure 1). The mannose binding ability of ConA was investigated after treatment with these hydrated ILs with different water content.<smiles>CCCC[N+](C)(C)C</smiles>

Tetrabutylammonium

[N4444]<smiles>C[P+](C)(C)[18OH]</smiles>

Tributyl-n-octylphosphonium [P4448]<smiles>C[P+](C)(C)P(C)(C)(C)C</smiles>

Tetrabutylphosphonium

[P4444]<smiles>C[N+](C)(C)CO</smiles>

Cholinium

[ch]<smiles>O=P([O-])(O)O</smiles>

Dihydrogen phosphate

[dhp]

$\mathrm{Br}^{-}$

Bromide

Figure 1. Structure of cations and anions for ionic liquids (ILs) used in the present study.

\section{Materials and Methods}

Concanavalin A (ConA) and bovine serum albumin (BSA) were purchased from SigmaAldrich Co. LLC. Mannotriose-modified BSA (mannotriose-BSA) was purchased from Dextra Laboratories, Ltd. 1-Ethyl-3-(3-dimethylaminopropyl)carbodiimide hydrochloride (EDC), N-hydroxysuccinimide (NHS), aminoethanol, guanidine hydrochloride (Gdn-HCl), choline chloride $(>98.0 \%)$, and other chemicals were purchased from Kanto Chemical Co., INC. Tetrabutylammonium bromide ( $>98.0 \%)$, tetrabutylphosphonium bromide $(>99.0 \%)$ and tributyl-n-octylphosphonium bromide $(>98.0 \%$ ) were purchased from Tokyo Chemical Industry Co, Ltd. Finally, phosphoric acid crystalline ( $>99.999 \%)$ was purchased from Sigma-Aldrich Co. LLC. ILs were synthesized by using an ion exchange resin, as previously described [23]. Synthesized ILs were characterized using electrospray mass spectrometry. Hydrated ILs were prepared by adding a calculated volume of water to thoroughly dried ionic liquids.

Lyophilized ConA dissolved in $10 \mathrm{mM}$ phosphate buffer ( $\mathrm{pH}$ 7.4) was separated by column (Sephadex G100), which has dextran chains. Lyophilized ConA was dissolved in buffer $(5 \mathrm{mg} / \mathrm{mL})$ and loaded on the column. Buffer was added to the column, then the eluted solution was collected by $1 \mathrm{~mL}$. After collecting $15 \mathrm{~mL}$ of eluted solution, additional solution was exchanged from buffer to $0.2 \mathrm{M}$ mannose solution. Inactivated ConA was eluted first, then retained active ConA at the resin surface was eluted after the addition of mannose solution. The concentration of eluted ConA was measured with UV-vis spectroscopy (U2450, Shimazu Corporation). After condensation, active ConA solution $(8.3 \mathrm{mg} / \mathrm{mL})$ was observed. Active ConA was prepared in hydrated [ch][dhp] to be mixed with three water molecules per ion pair $(1.75 \mathrm{mg} / \mathrm{mL})$. After diluting the solution 100 times with buffer, the mannose binding ability was measured using the biolayer interferometry method. 
Purchased lyophilized ConA was dissolved at $5 \mathrm{mg} / \mathrm{mL}$ in hydrated ILs and buffer as a reference. Hydrated ILs were prepared in ratios of 3,7, and 15 water molecules per ion pair. Lyophilized ConA was mixed overnight with prepared hydrated ILs and buffer at room temperature. After dilution with buffer, the carbohydrate-binding ability of ConA was investigated by biolayer interferometry.

Mannose recognition and binding ability of ConA treated in hydrated ILs were analyzed after 100 times dilution with a buffer solution using biolayer interferometry (BLItz, Fortebio Molecular Devices Ltd., Fremont, CA, USA). Mannotriose-BSA and BSA were immobilized on an AR2G sensor chip. The binding experiment was performed by first dipping the sensor in a sample, which was diluted 100 times with buffer, for $120 \mathrm{~s}$ to measure association. This was followed by dipping the sensor in buffer for $180 \mathrm{~s}$ to measure dissociation. The sensorgrams obtained with buffer containing ILs without ConA was used as reference and subtracted from the sample sensorgrams.

\section{Results}

\subsection{Separation of Lyophilized ConA and the Mannose Binding Ability}

Figure 2 insert shows absorbance at $280 \mathrm{~nm}$, which corresponds to the protein concentration in the eluent. The chromatogram showed two peaks: the first peak appeared shortly after injecting the sample into the column and corresponds to ConA with poor affinity for dextran. The second peak in the chromatogram was observed after the addition of a mannose solution. Based on the areas under the peaks, it was estimated that lyophilized ConA contains approximately $20 \%$ of denatured ConA with poor affinity for dextran (glucose component). These eluted peaks were collected, and the concentration of the solution was adjusted to $38 \mu \mathrm{g} / \mathrm{mL}$. Then, the respective mannose binding abilities were measured using biolayer interferometry. It was observed that mannose binding ability differed here; low mannose binding activity was observed in the fraction corresponding to the first peak, and it showed no dextran binding ability (Figure 2, blue line). On the other hand, relatively high mannose binding ability was observed in the fraction corresponding to the second peak, and it showed strong affinity to dextran (Figure 2, green line). The mannose binding ability in first peak was about $40 \%$ that of the fraction from the second peak.

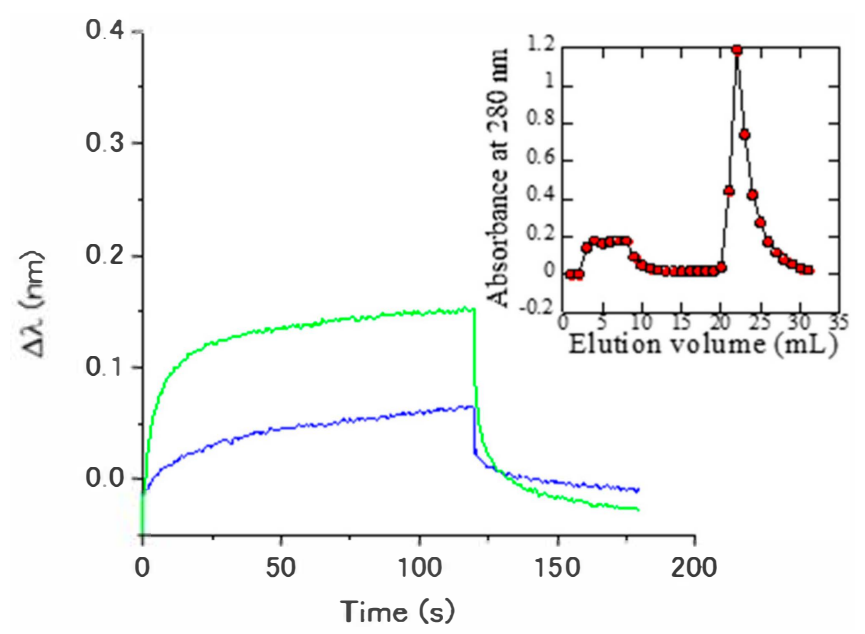

Figure 2. Mannose binding ability of separated ConA eluent in the first peak (blue) and in the second peak (green). Insert: Absorbance change in eluted solution of ConA.

\subsection{Mannose Binding Ability of ConA, After Treatment in Hydrated ILs}

3.2.1. Effect of the Water Content of Hydrated ILs on the Mannose Binding Ability of ConA

Figure 3 shows the mannose binding ability of ConA after treatment with hydrated [ch][dhp] mixed with 3, 7, and 15 water molecules per ion pair and that in buffer. ConA was dissolved in hydrated [ch][dhp], then diluted 100 times with buffer to measure mannose 
binding ability. A significant increase (around twofold) in the mannose binding ability was observed in samples treated in hydrated [ch][dhp] with three water molecules per ion pair, compared with samples dissolved in buffer. Samples treated with hydrated [ch][dhp] mixed with seven water molecules per ion pair showed a mannose binding ability similar to that of lyophilized ConA dissolved directly in buffer. Samples treated with hydrated [ch][dhp] containing 15 water molecules per ion pair showed lower mannose binding ability. It was suggested that the high salt solution would have a negative effect on the renaturation of ConA. A negative correlation was found between the increase in mixing ratios of water molecules per ion pair and the mannose binding ability. No increase in the mannose binding ability was observed when lyophilized ConA was directly dissolved in buffer containing [ch][dhp] (at a concentration equivalent to the treatment with [ch][dhp]), mixed with three water molecules and then diluted 100 times with buffer. Furthermore, no increase in the binding ability shortly after dissolution of ConA in hydrated [ch][dhp] was found. It was suggested the incubation time is one of the important factors of renaturation in hydrated [ch][dhp]. Effect of water contents on the binding ability were observed similar with hydrated [ch][dhp] after treatment with other hydrated ILs composed of ammonium and/or phosphonium cation and dhp anion. Increase in the mannose binding ability was observed after treatment in hydrated ILs with three water molecules per ion pair. The mannose binding ability decreased with an increase in water content. In the case of hydrated ILs composed of bromide anions, no increase in mannose binding ability was observed, regardless of the water content (Figure S1). The observed mannose binding responses after treatment with hydrated ILs were sometimes found to be unstable except for [ch][dhp] treatment. Ammonium and phosphonium ILs were occasionally effective on the sensor chip even without ConA. Further investigation was proceeded by using hydrated [ch][dhp].

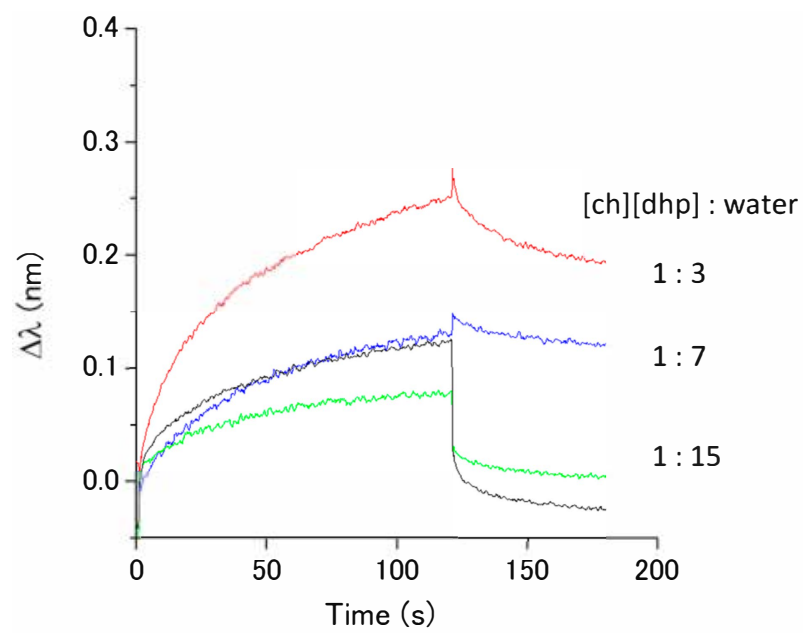

Figure 3. Mannose binding ability of lyophilized ConA dissolved in buffer (black), and ConA treated in hydrated [ch][dhp] mixed with 3 (red), 7 (blue), and 15 (green) water molecules per ion pair.

3.2.2. Assessment of the Mannose Binding Ability of Active, Inactive, and Reactivated Forms of ConA

After treatment of lyophilized ConA with hydrated [ch][dhp] containing three water molecules per ion pair, ConA was loaded on the dextran column as described in Section 3.1. The chromatogram showed two peaks. They were separately collected, and the concentration was adjusted to ensure the same concentration in the two fractions. The mannose binding ability was measured by biolayer interferometry (Figure 4, red line). The black line represents mannose binding responses based on each component without treatment with hydrated [ch][dhp]. The response of eluted solution in the first peak (low mannose binding ConA) with treatment showed an increase of about 3.3 times in comparison to that without 
the treatment. In the case of the second peak (higher mannose binding ConA), the increase in the response with treatment was about 1.8 times compared to that without treatment.
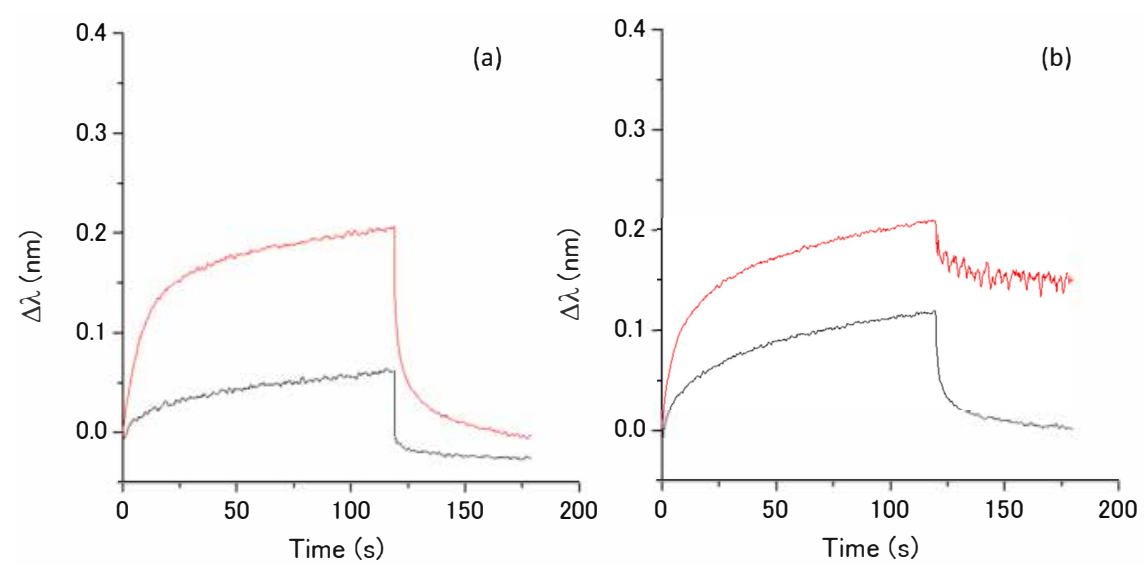

Figure 4. Mannose binding ability of ConA eluent from the first peak (a) and in second half peak (b). Each sensorgram shows binding change before (black) and after (red) treatment with hydrated [ch][dhp].

\section{Discussion}

Commercially available lyophilized ConA contains two different components: one shows low affinity for binding to glucose and mannose, and the other displays high binding affinity. Increase in the mannose binding ability of ConA depends on the water content, which suggests that renaturation of low-binding ConA was affected by water states (free water or binding water) in hydrated ILs. Furthermore, the anion structure of hydrated ILs influenced the recovery of the binding ability. Hydrated ILs composed of dhp anion (a kosmotropic ion) showed an effective increase in mannose binding ability. On the other hand, the effect of the cation was not so obvious. Similar results with [ch][dhp] were observed in ammonium and phosphonium ILs with dhp anion. It was considered that the hydroxyl group in the choline cation was not a key structure for the renaturation of lyophilized ConA. These conditions suggest that hydrated ILs, which contain almost no free water and have high hydrogen bonding ability, have the potential to aid the renaturation of inactive component in lyophilized ConA. Authors have reported that the hydration state in hydrated ILs depends on the water content using different methods such as water activities analysis, DSC, NMR measurements, and molecular simulation investigations [24-27]. Water molecules in hydrated ILs behave not as free water but bound water when the water content is below seven water molecules per ion pair [24]. Considering the decline in mannose binding ability with increasing water content, existence of free water is perhaps unnecessary for the stable dissolution and restructuring of ConA.

After treatment of lyophilized ConA in hydrated [ch][dhp] with three water molecules per ion pair, an increase in mannose binding ability was observed. Both components of ConA, those with low and high mannose binding ability, showed an increase in mannose binding ability. The increased ratio of this binding ability was different; the mannose binding ability of these two components became similar after treatment with hydrated [ch][dhp] containing three water molecules per ion pair. Low mannose binding ConA showed a greater increase ratio than high mannose binding ConA. Interestingly, improvement in glucose binding ability was not observed. It was suggested that the glucose binding site was not affected by the restructuring in hydrated [ch][dhp]. ConA can recognize and bind to mannose and glucose among carbon hydrate chains. Originally, the dextran binding ability of ConA was lower than its mannose binding ability [28]. This might be related to the position of the binding site and/or binding manner to glucose.

The observed increment in total mannose binding ability after treatment with hydrated [ch][dhp] (Figure 2, red line) was compared with that calculated using the increment of each response based on the separated ConA (Figure 4). As seen in the chromatogram 
in Figure 2 insert, lyophilized ConA consisted of 20\% low mannose binding ConA and $80 \%$ high mannose binding ConA. ConA without treatment showed a total binding value $(\Delta \lambda)$ of 0.125 at $120 \mathrm{~s}$ (Figure 3, black line). The estimated binding values of low and high mannose binding ConA were 0.025 and 0.100, respectively. Estimated increases in the binding value after treatment were 0.083 for low mannose binding ConA and 0.180 for high mannose binding ConA. These were calculated by using increase rates of 3.3 and 1.8 times, respectively. When adding these values, the total increase in the binding value was estimated to be 0.263 . The measured value (Figure 3) was 0.251 . These values showed reasonable correlation.

\section{Conclusions}

Purchased lyophilized ConA containing low mannose binding ConA showed an increase in its mannose binding ability after dissolution in hydrated ILs composed of dhp anions. The increase in the mannose binding activity was dependent on the water content in the hydrated ILs. As the water content increased, the mannose binding ability decreased. Water state in hydrated ILs might be an important factor for the renaturation of inactivated ConA. Overall, our results support the use of hydrated ILs, especially [ch][dhp], as a novel treatment solvent for renaturation of lyophilized proteins that may be damaged by the procedure. This simple procedure is expected to be a valid and feasible method for further development of protein applications.

Supplementary Materials: The following are available online at https: / / www.mdpi.com/2076-341 7/11/1/57/s1, Figure S1: Mannose binding responses of ConA which had dissolved in buffer and hydrated ILs followed by dilution with buffer.

Author Contributions: Data curation, R.F.; project administration, K.F.; writing-original draft preparation, R.F., K.F.; writing — review and editing, K.F.; funding acquisition, K.I., K.F. All authors have read and agreed to the published version of the manuscript.

Funding: This research was supported by a Grant-in-Aid for Scientific Research from the Japan Society for the Promotion of Science (KAKENHI, No. 17H01225 and 18H01719).

Data Availability Statement: Please refer to suggested Data Availability Statements in section "MDPI Research Data Policies" at https://www.mdpi.com/ethics.

Conflicts of Interest: The authors declare no conflict of interest.

\section{References}

1. Matejtschuk, P. Lyophilization of proteins. Methods Mol. Biol. 2007, 368, 59-72. [CrossRef] [PubMed]

2. Liu, B.; Zhou, X. Freeze-drying of proteins. Methods Mol. Biol. 2015, 1257, 459-476. [CrossRef] [PubMed]

3. Cevasco, G.; Chiappe, C. Are ionic liquids a proper solution to current environmental challenges? Green Chem. 2014, 16, 2375-2385. [CrossRef]

4. Handy, S.T. Grignard reactions in imidazolium ionic liquids. J. Org. Chem. 2006, 71, 4659-4662. [CrossRef] [PubMed]

5. Ohno, H.; Fukaya, Y. Task Specific Ionic Liquids for Cellulose Technology. Chem. Lett. 2009, 38, 2-7. [CrossRef]

6. Kathiresan, M.; Velayutham, D. Ionic liquids as an electrolyte for the electro synthesis of organic compounds. Chem. Commun. 2015, 51, 17499-17516. [CrossRef]

7. Itoh, T. Ionic Liquids as Tool to Improve Enzymatic Organic Synthesis. Chem. Rev. 2017, 117, 10567-10607. [CrossRef]

8. D'Arrigo, P.; Cerioli, L.; Chiappe, C.; Panzeri, W.; Tessaro, D.; Mele, A. Improvements in the enzymatic synthesis of phosphatidylserine employing ionic liquids. J. Mol. Catal. B Enzym. 2012, 84, 132-135. [CrossRef]

9. Clarke, C.J.; Tu, W.C.; Levers, O.; Bröhl, A.; Hallett, J.P. Green and Sustainable Solvents in Chemical Processes. Chem. Rev. 2018, 118, 747-800. [CrossRef]

10. Maton, C.; de Vos, N.; Stevens, C.V. Ionic liquid thermal stabilities: Decomposition mechanisms and analysis tools. Chem. Soc. Rev. 2013, 42, 5963-5977. [CrossRef]

11. Wasserscheid, P.; Welton, T. (Eds.) Ionic Liquids in Synthesis; Wiley-VCH: Weinheim, Germany, 2007.

12. Welton, T. Ionic liquids: A brief history. Biophys. Rev. 2018, 10, 691-706. [CrossRef] [PubMed]

13. Kohno, Y.; Deguchi, Y.; Ohno, H. Ionic liquid-derived charged polymers to show highly thermoresponsive LCST-type transition with water at desired temperatures. Chem. Commun. 2012, 48, 11883-11885. [CrossRef] [PubMed]

14. Ichikawa, T.; Fujimura, K.; Yoshio, M.; Kato, T.; Ohno, H. Designer lyotropic liquid-crystalline systems containing amino acid ionic liquids as self-organisation media of amphiphiles. Chem. Commun. 2013, 49, 11746-11748. [CrossRef] [PubMed] 
15. Schindl, A.; Hagen, M.L.; Muzammal, S.; Gunasekera, H.A.D.; Croft, A.K. Proteins in Ionic Liquids: Reactions, Applications, and Futures. Front. Chem. 2019, 7. [CrossRef] [PubMed]

16. Attri, P.; Venkatesu, P.; Kumar, A. Water and a protic ionic liquid acted as refolding additives for chemically denatured enzymes. Org. Biomol. Chem. 2012, 10, 7475-7478. [CrossRef] [PubMed]

17. Yamaguchi, S.; Yamamoto, E.; Mannen, T.; Nagamune, T.; Nagamune, T. Protein refolding using chemical refolding additives. Biotechnol. J. 2013, 8, 17-31. [CrossRef] [PubMed]

18. Fujita, K.; Nakano, R.; Nakaba, R.; Nakamura, N.; Ohno, H. Hydrated ionic liquids enable both solubilisation and refolding of aggregated concanavalin A. Chem. Commun. 2019, 55, 3578-3581. [CrossRef]

19. Fujita, K.; Kajiyama, M.; Liu, Y.; Nakamura, N.; Ohno, H. Hydrated ionic liquids as a liquid chaperon for refolding of aggregated recombinant protein expressed in Escherichia coli. Chem. Commun. 2016, 52, 13491-13494. [CrossRef]

20. Idil, N.; Percin, I.; Karakoc, V.; Yavuz, H.; Aksoz, N.; Denizli, A. Concanavalin A immobilized magnetic poly(glycidyl methacrylate) beads for prostate specific antigen binding. Colloids Surf. Bbiointerfaces 2015, 134, 461-468. [CrossRef]

21. Fujita, K.; Sanada, M.; Ohno, H. Sugar chain-binding specificity and native folding state of lectins preserved in hydrated ionic liquids. Chem. Commun. 2015, 51, 10883-10886. [CrossRef]

22. Fujita, K.; Ohno, H. Enzymatic activity and thermal stability of metallo proteins in hydrated ionic liquids. Biopolymers 2010, 93, 1093-1099. [CrossRef] [PubMed]

23. Fujita, K.; MacFarlane, D.R.; Forsyth, M.; Yoshizawa-Fujita, M.; Murata, K.; Nakamura, N.; Ohno, H. Solubility and Stability of Cytochrome c in Hydrated Ionic Liquids: Effect of Oxo Acid Residues and Kosmotropicity. Biomacromolecules 2007, 8, 2080-2086. [CrossRef] [PubMed]

24. Ohno, H.; Fujita, K.; Kohno, Y. Is seven the minimum number of water molecules per ion pair for assured biological activity in ionic liquid-water mixtures? Phys. Chem. Chem. Phys. 2015, 17, 14454-14460. [CrossRef] [PubMed]

25. Fujita, K.; Nikawa, Y.; Ohno, H. Cold crystallisation behaviour of water molecules in ionic liquids as a screening method to evaluate biocompatibility of the hydrated ionic liquids. Chem. Commun. 2013, 49, 3257-3259. [CrossRef] [PubMed]

26. Nikawa, Y.; Fujita, K.; Ohno, H. Quantitative assessment of kosmotropicity of hydrated ionic liquids by nuclear magnetic resonance. Phys. Chem. Chem. Phys. Pccp 2017, 19, 8148-8151. [CrossRef]

27. Nikawa, Y.; Tsuzuki, S.; Ohno, H.; Fujita, K. Hydration States of Cholinium Phosphate-Type Ionic Liquids as a Function of Water Content*. Aust. J. Chem. 2019, 72, 392-399. [CrossRef]

28. Oda, Y.; Kasai, K.; Ishii, S. Studies on the specific interaction of concanavalin A and saccharides by affinity chromatography. Application of quantitative affinity chromatography to a multivalent system. J. Biochem. 1981, 89, 285-296. [CrossRef] 